\title{
Research on Micro-lesson Resource Service Platform and Resources Scheduling Model
}

\author{
XU Yabin ${ }^{1,2}$ PENG Hongen ${ }^{1}$ \\ 1.Computer Institute of Beijing Information Science and Technology University, \\ Beijing, 100101 \\ 2. Beijing Key Laboratory of Internet Culture and Digital Dissemination \\ Research, Beijing Information Science \&Technology University, Beijing, 100101) \\ yabin_xu@bistu.edu.cn, peng3307165@sina.com
}

\begin{abstract}
In order to meet the need of rapid growth of the micro-class resources, it is urgently need to provide a highly reliable and cost-effective hosting platform. The architecture design of micro-lesson resource service platform based on PaaS pattern and the microlesson resource scheduling model based on MapReduce are given in this paper. Comparative experiment results show that, the three indicators of average response time, average load rate of server, and standard deviation of server load rate are better than that of traditional server clustering and OpenStack resource scheduling algorithm.
\end{abstract}

Keywords: PaaS; micro-lesson resource service platform; MapReduce; resource scheduling model

\section{Introduction}

Micro-lesson takes video as main carrier, record whole process of a wonderful teaching activity undertaken by a teacher around a knowledge point or teaching link in the classroom teaching. [1] The core of a micro-lesson is micro-video, also includes other supplementary teaching resources associated with this knowledge point, such as microcourseware, micro-comment, micro-teaching-plan, micro-reflection, micro-exercises, micro-test, etc. They jointly create a thematic knowledge unit with certain organization relationship and presentation pattern. Therefore, the micro-lesson is not only distinct from any traditional type of teaching resources such as instruction video, teaching case, courseware and others, but also a new type of teaching pattern which extended and developed on the basis of them.

Micro-lesson is an extension and expansion of traditional classroom teaching. It is a new teaching pattern and teaching philosophy combined with the Internet. Its features are as follows:

1. Short course time: the best time length of a micro-lesson video is about 4-5 minutes, usually not more than 10 minutes. Compared with the traditional classroom, students may pay attention to learning and avoid wasting study time in the learning process of the micro-lesson video.

2. Prominent theme: in micro-lesson teaching process, each instructional video usually explains only one knowledge point. The theme of a course is distinctive. Students can easily and quickly find what they want to learn, thus making learning more targeted.

3. Refining teaching content: since the time of a micro-lesson is short, the curriculum must be compact, excellence, quality and efficient.

4. Strong interest: micro-lesson can't be explained boringly, should have a strong interest, can attract learners to be enthusiasm and initiative.

5. Abundant supporting materials: only micro-video is often not enough, it also requires micro-courseware, micro-comment, micro-reflection, micro-exercises, micro-testing, 
etc. Through mutual setting off, cooperating each other, we can achieve the desired teaching effect.

6. Course video is posted to the web, and students can make full use of high-quality teaching resource at any time and any place.

Micro-lesson resource is one kind of most popular resource developed and applied in education. Its growing fast, urgently need a highly reliable and cost-effective hosting platform. Meanwhile, the micro-lesson resource service is also an important resource on "public service platform of educational resource". Therefore, the study and design of micro-lesson resource service platform, not only can effectively hosting and support micro-lesson resource service, but also is an active exploration for building the public service platform of educational resource. It has an important reference value for the construction of public service platform of educational resource.

\section{The Situation of Micro-Lesson Resource Service Platform}

Because the micro-lesson mode itself is of flexibility and interactivity, as well as in line with the current development of IT technology and applications, micro-lesson teaching pattern has caused more and more educators' attention. It will develop more rapidly and apply widely in the future. In this case, in order to support micro-lesson teaching effectively, researching and designing a micro-lesson resource service platform with superior performance and reliability is of important significance and great value.

Currently in technology, micro-lesson provides services through traditional "web page+ background server"[2]. Although adopting server cluster technology and network storage technology can improve service's reliability and stability to a certain extent, but it can't make resource shared, efficient and rationally used. There is a serious waste of resource.

In general, whether in domestic or foreign, the development and construction about the micro-lesson resource service platform are still in research and exploration stage. Large scale of application and practice is still blank. To this end, we give a micro-lesson resource service platform designed with the idea of PaS, as well as a distributed parallel scheduling model based on MapReduce. The platform can share hardware and software resource maximize. It not only can allocate and recycle resources according to the need, to ensure efficient use of resources, but also can effectively meet the need of different sizes of micro-lesson teaching applications.

\section{Design of Micro-lesson Resource Service Platform Based on PaaS}

\subsection{PaaS Service Mode}

Cloud computing is a large-scale distributed computing mode based on internet, it is a new service delivery and usage pattern. It releases involved hardware and system software as the pattern of service and provides safely and quickly data storage and computing service [3].

There are three kinds of cloud computing service modes: Infrastructure as a Service (IaaS), Platform as a Service (PaaS), Software as a Service (SaaS). They are shown in Figure 1.

We can see from Figure 1, PaaS builds on the found of virtualized resource service provided by IaaS. It can provide downward transparent access to hardware equipment, such as server, network storage device, and switch etc. It supports upward centralized software deployment on platform service layer for software service provider, and provides software running service and data access service for tenants. Thus, PaaS becomes the most important cloud computing service mode. 


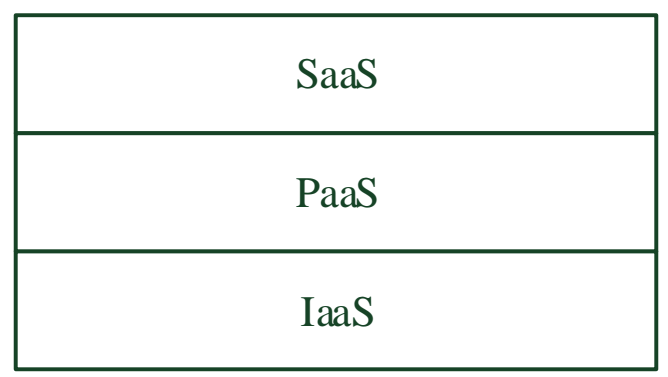

Figure 1. Cloud Computing Model

On service pattern, by customizing the hardware and software resource, PaaS can provide users with specific software operating environment. To publishing applications, users only need to upload developed applications to the PaaS platform. The more valuable point of $\mathrm{PaaS}$ platform is that it provides dynamic resource scheduling pattern. By monitoring the traffic of application, the PaaS platform can dynamically allocate resources for specific applications, so that the resources can automatically adapt to traffic changes.

PaaS mainly provides application development, testing and application hosting service to users. Among them, the development and testing cloud service platform provides the appropriate hardware, software resources and running environment for developers in the form of Web API. However, application hosting cloud service platform deploys resources and applications submitted by privileged users to open platform, it generates different application instance for different user during operation and provides the appropriate running environment.

\subsection{The Design of Micro-Lesson Resource Service Platform}

Micro-lesson resource service platform belongs to the application hosting cloud service platform. The application hosting cloud service platform is different from traditional hosting service. The latter's hardware resource is fixed and can't be dynamically expanded, even limited to a specific server. If the application's demand for resources exceeds the amount of agreed allocation, it will lead to run slowly or even crash. The former's resource is elastic, allocated by monitoring the resource request. It can increase or decrease the supply of resource, thereby improve resource utilization efficiency. In addition, it provides load balancing, multiple-copy deployment, task migration mechanism to ensure high availability of application.

The architecture of micro-lesson resource service platform is shown as Figure 2.

$\mathrm{PaaS}$ provides micro-lesson resource service to users in the form of Web application. Micro-lesson resource provider can submit micro-lesson resources to the platform after the adoption of certification. Platform administrator establishes link to corresponding service component in accordance with the form of micro-lesson component, and submit the application of resource to the platform. Micro-lesson resource user directly accesses micro-lesson resource through a Web page.

Resource request monitoring module determines the number of resource scheduling through monitoring the number of HTTP request (application traffic) of Web application, and adjust timely. Resource scheduling module is not only responsible for resource allocation of application request, but also responsible for overall scheduling of software and hardware resource. Load balancing module is mainly used to allocate server's requests to the physical equipment in balance. If an application server is abnormal load or error occurred, the service can be migrated to other server by task migration module. 


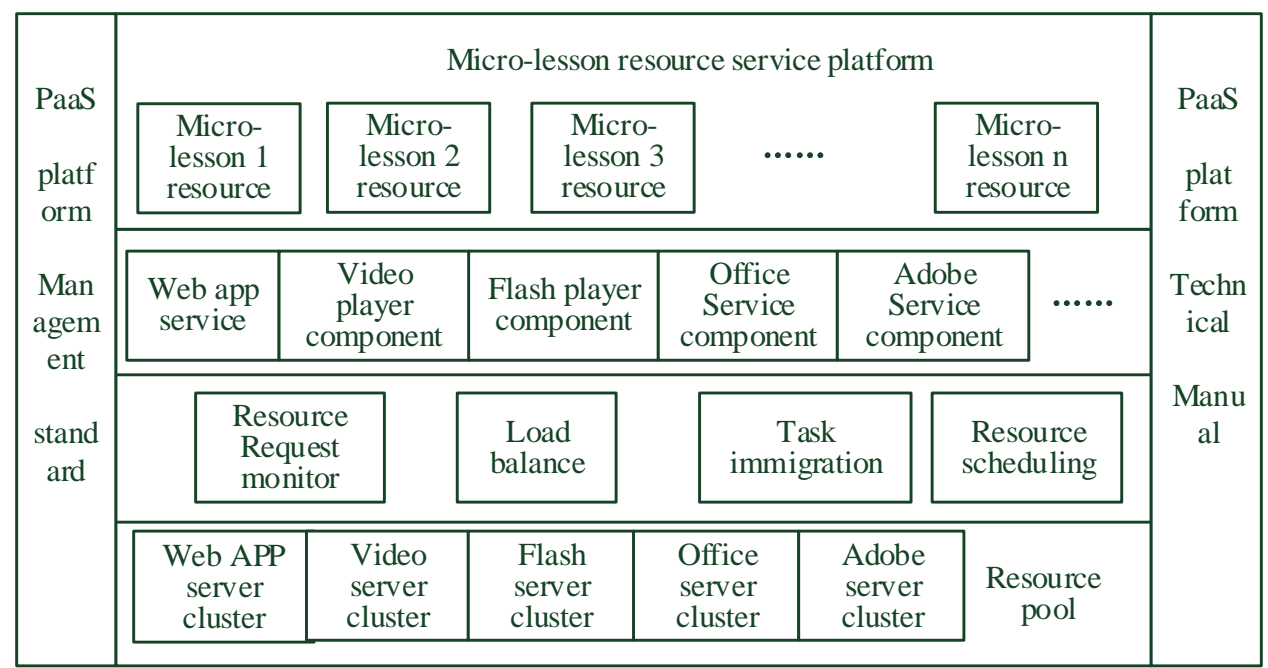

Figure 2. The Architecture of Micro-Lesson Resource Service Platform

Specific application server cluster is established based on the possible resource consumption. During operation, it can allocate and adjust resource according to the specific circumstances of the resources request.

\section{PaaS Resource Scheduling Model}

Resource scheduling capability is critical important for PaaS platform, the merits of its resource scheduling capability directly determine the operating efficiency of PaaS.

Currently, the resource scheduling algorithm described by vast amount of literatures is about IaaS, IaaS cloud resource scheduling algorithm is divided into two classes:

(1) Resource allocation and scheduling algorithm to the maximum utilization of resources as the goal in the premise of ensuring the performance. Such algorithms realize consult between users and providers of resource through SLA resource allocator, and realize optimal allocation of resources.

(2) Resource allocation and scheduling algorithm to the lowest energy consumption as the goal. This kind of algorithm emphatically considers the demand for energy consumption. When resource request is less, let as much as possible servers in a dormant state.

The resource scheduling of PaaS is different from that of IaaS, the main differences are as follows [5]:

(1) The frequency of PaaS resource scheduling is much smaller than that of IaaS resource scheduling. Generally, users' IaaS resource needs is less variable in short time. But In PaaS, the resources needs of Web applications may change frequently in a short time.

(2) The magnitude of IaaS resource scheduling is much smaller than the magnitude of PaaS resource scheduling. Generally, users' IaaS resource needs change often slowly, increment or decrement are relatively small. But in PaaS, the resources needs of Web applications may increase or decrease sharply.

(3) Generally, Resource scheduling in IaaS is often based on virtual machines, the granularity of resource scheduling is large, and the cost of resource scheduling is relatively high. While in PaaS, the resource usage of a Web application instance is less, and the cost of resource scheduling is lower. 
Therefore, PaaS cloud resource scheduling algorithm is difficult to directly follow the current research results about IaaS cloud resource scheduling. It must be designed for the actual situation of PaaS. However, it's not realistic to self-develop and design. As there is no support of basic cloud platform, it is difficult to ensure the performance and stability. So, PaaS must build on the existing open source cloud computing infrastructure.

Currently, there are two widely accepted open source cloud computing framework with the best performance and stability, one is OpenStack, another is MapReduce.

OpenStack is the most widely used open source cloud computing management platform. You can get a variety of services by calling various API, integrate on the framework, realize a IaaS which is similar to Amazon EC2 and S3. OpenStack consists of two main modules: Nova and Swift. Nova is a virtual server deployment and business computing module developed by NASA, and Swift is a distributed cloud storage module which is developed by Rackspace.

Hadoop is an open source cloud computing platform, and its core technology is HDFS and MapReduce. Wherein, HDFS provides storage services for massive data, MapReduce not only can be used for fast parallel computing, but also can efficiently carry out multitask scheduling. MapReduce encapsulates functional components such as load balancing, fault-tolerant processing, parallel processing, localization computing, and provides the corresponding calling interface [6-7].

Through the analysis and comparison, we can find that, OpenStack is mainly a resource scheduling and management platform based on virtual machine. While MapReduce mainly provide task (application) oriented resource scheduling and parallel processing. So, the MapReduce is easier to implement application-oriented elastic resource scheduling and management. Compared with the virtual machine scheduling of the OpenStack platform, the PaaS design based on the MapReduce platform can be more reasonable to use the network resource and save time. Finally, we determine that Implement of resources scheduling and management is based on MapReduce model.

The micro-lesson resource scheduling and management model based on MapReduce is shown in Figure 3.

The working process is as follows:

(1) Resource request monitoring module will convey the request of resource to control module Master by monitoring the requests. Master allocates the number of Map nodes $\mathrm{M}$, and the number of Reduce nodes $\mathrm{N}$ according to the resource request, and call the idle nodes in application server cluster to read the data which the user request for.

(2) According to the prior written micro-lesson resource reading program, create $\mathrm{m}$ copies of the program and issue to each Map node. According to the prior written micro-lesson resource merge program, create $\mathrm{n}$ copies of the program and issue to each Reduce node.

(3) Each node that is assigned a Map task executes the corresponding program to read the relevant micro-lesson resource data fragment, and parses key/value pair from the data segment. The key/value key pair is passed to the pre-defined Map function, then the Map function generates and outputs intermediate key/value key pair and store them in the cache space.

(4) The key/value pair in buffer space is divided into $\mathrm{n}$ regions by the partitioning function, and then periodically written to each region. The corresponding storage location information is transmitted back to Master, Master is responsible for transmitting the information to the task in Reduce node. 


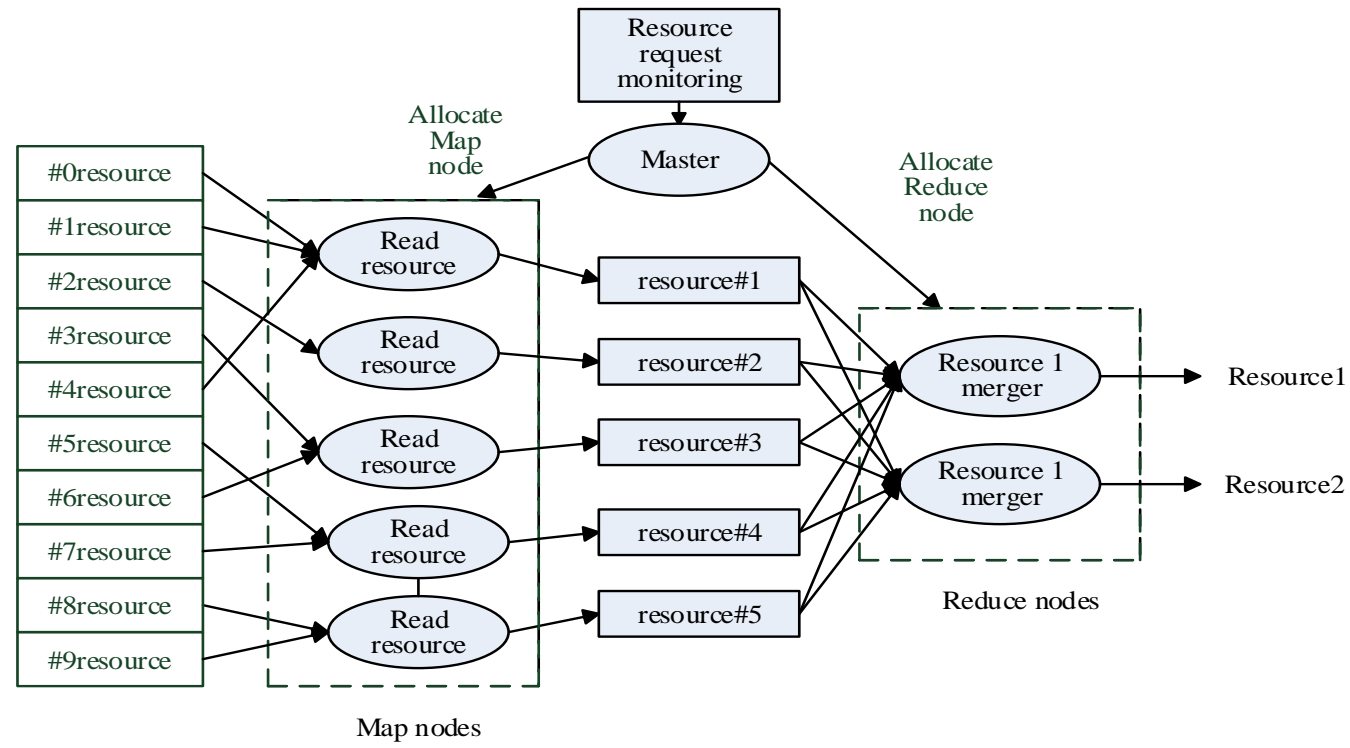

Figure 1. Cloud Computing Model

(5) When the task in the Reduce node receives the feedback information from the Master program, use the RPC interface to read all of the intermediate data in each cache space, and then sort the key, so that the data fragments with the same key values are aggregated together.

(6) After traverse sorted intermediate data, the tasks in the Reduce nodes pass each key value and its associated intermediate value collection to the customized Reduce function. The function's output is appended to micro-lesson resource data set of its partition.

(7) When all the Map and Reduce tasks are completed, the Master will wake up the Web application program and return requested complete micro-lesson resources.

(8) It can be seen from Figure 3 and its working process, the number of computing nodes and tasks for reading and merging micro-lesson resource are completely determined and allocated on demand.

\section{Experimental Results and Comparative Analysis}

In order to verify the application effect of the micro-lesson resource service platform based on MapReduce, we respectively use 5 servers and a switch in the laboratory to build a server cluster environment, OpenStack platform and the micro-lesson resource service platform proposed in this paper to carry out the contrast experiment. In the process of experiment, the hardware and software of the server cluster, OpenStack platform and MapReduce platform are consistent, and the scale of resource request is also consistent.

In the experiment, we deploy the control function entity on a control server, using 4 servers as APP servers to deploy different classes of micro-lesson resources. Among them, the control server has the configurations: $2 \times 8$-core Intel Xeon E5-2670 processor, 32GB RAM and CentOS 6.5 operating system. Other servers have the configurations: 1×8-core Intel Xeon E5-2670 processor, 16GB RAM and CentOS 6.5 operating system. We analyze the performance of the platform through the statistical data of the system running on each platform.

We select the average response time of the system, the average load rate of the server and the standard deviation of server load rate as the main indexes in the experiment. System response time is the elapsed time in the process from users submitting requests to the application system displaying the results of this operation in the form of user can 
perceive. Response time reflects resource scheduling capability of a system or platform in the case of large amount of tasks. Due to the uncertainty of the user's task request and the operating environment, we use the average response time to measure the performance of the platform.

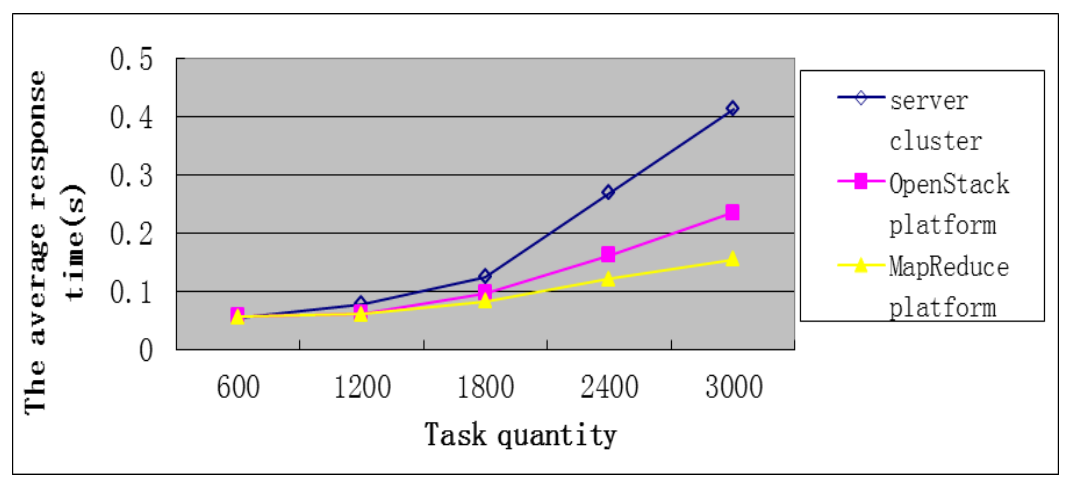

Figure 4. The Average Response Time of System

Figure 4 described the changes in the average response time of the three platforms in the case of a constant increase of tasks. It can be seen, due to the use of multi replica deployment and distributed parallel scheduling model, the micro-lesson resource service platform based on MapReduce is better than the other two platforms when tasks are constant increasing. Especially in the case of large amount of tasks, it can make a quick response for user's request.

We still record the average load rate of the physical server and the standard deviation of server load rate in the three platforms. The higher load rate of server is, indicating the higher utilization of resources is, and the fewer resources wasted phenomenon is. Standard deviation also known as mean square deviation, it is arithmetic square root of variance, which describes the deviation degree between variables and means. In this experiment, the smaller the standard deviation is, indicating the closer load of each server is, that is the more balanced load. The average load rate of server and standard deviation of server load rate in each platform is shown in Table 1.

Known from the data in Table 1, MapReduce model has highest load rate, may better schedule and manage resource and use resource rationally. And the standard deviation of server load rate in MapReduce platform is smaller, the effect of load balance is the best.

Table 1. Average Load Rate and Standard Deviation of Server

\begin{tabular}{lcc}
\hline \multicolumn{1}{c}{ Platform } & $\begin{array}{c}\text { The average load } \\
\text { rate of server }\end{array}$ & $\begin{array}{c}\text { The standard deviation of } \\
\text { server load rate }\end{array}$ \\
\hline Server cluser & $72.95 \%$ & 0.067 \\
OpenStack model & $75.33 \%$ & 0.033 \\
MapReduce model & $76.75 \%$ & 0.016 \\
\hline
\end{tabular}

\section{Conclusions}

Using advanced cloud computing technology to build a large open service platform of micro-lesson resource has important strategic significance in promoting the reform of teaching pattern in the information environment, promoting the construction of "three through two platform". As a subset of the public services platform of educational resource, we hope that, through the research and design of micro-lesson resource service platform, provide reference for the construction of public service platform of educational resources, on one hand. On the other hand, the excellent teaching resources can be released to more students and make the micro-lesson resources get a wider range of 
applications. so that, bring the further improvement of the teaching effect and the quality of education, the effective elimination of the unbalance distribution of educational resources and unequal access to education, and promote fairness in education.

As we used the resource scheduling model based on MapReduce, it can provide the high flexibility and high scalability for the micro-lesson service platform. Developers do not need to pay attention to the scale of the underlying hardware and processing capability. The platform will automatically adjust the service scale based on the application load and maximize the utilization of resource.

Comparative experimental results show that, the micro-lesson resource service platform and the micro-lesson resource scheduling model we designed are better than traditional server cluster and OpenStack resource scheduling algorithm in the average response time of the system, the average load rate of the server and the standard deviation of server load rate.

\section{Acknowledgements}

This work was supported by the National Natural Science Foundation of China(Grant No.61370139), Beijing Key Laboratory of Internet Culture and Digital Dissemination Research (ICDD201506), Beijing "three through two platform" standard demonstration project (BJW 201401) and Beijing collages innovation team building and teacher occupation development program(IDHT20130519)

\section{References}

[1] Hu Tiesheng, Huang Mingyan, Li Min. The three stages and enlightenment of the development of Chinese micro-lesson[J], Distance Education Journal, 4 (2013), 36-42.

[2] Zhang Yichuan. Qian Yangyi. Progress in the construction and application of the micro-lesson at China and abroad[J], Distance Education Journal, 6 (2013), 26-33.

[3] Gao Yunxiang, Hao Shukui, Cheng Xiang. Analysis of the key technologies of PaaS in private cloud[J], Designing Techniques of Post and Telecommunications 7 (2014).

[4] Wen Jianrong. Research and implement of Java Web application scheduling mechanism in PaaS cloud platform[D], Beijing University of Posts and Telecommunications, 12 (2012).

[5] Xu Peng, Zhang Yanjiang, Su Sen. Resource scheduling technology of PaaS cloud platform[J], Journal of Huazhong University of Science and Technology, vol.41, z2 (2013).

[6] Guo Lijiao. Research on virtual resource scheduling in cloud environment [D], Taiyuan University of Technology, 5 (2014).

[7] Wu Haiyan. Research on resource scheduling algorithm based on cloud computing environment [D], Jiangxi University of Science and Technology, 6 (2012).

\section{Authors}

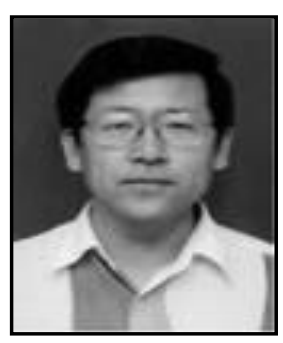

XU Yabin. Professor, the main research directions for cloud computing and big data, social networks, future networks. 